This item was submitted to Loughborough's Research Repository by the author.

Items in Figshare are protected by copyright, with all rights reserved, unless otherwise indicated.

\title{
Food-to-mealtime associations influence food selection in a UK-based sample
}

PLEASE CITE THE PUBLISHED VERSION

https://doi.org/10.46439/nutrition.1.004

PUBLISHER

ProBiologists LLC

VERSION

VoR (Version of Record)

LICENCE

CC BY 4.0

\section{REPOSITORY RECORD}

McLeod, Chris, Lewis James, and Gemma Witcomb. 2020. "Food-to-mealtime Associations Influence Food Selection in a Uk-based Sample”. Loughborough University. https://hdl.handle.net/2134/13072133.v1. 


\title{
Food-to-mealtime associations influence food selection in a UK-based sample
}

\author{
McLeod CJ, James LJ, Witcomb GL*
}

School of Sport, Exercise and Health Sciences, Loughborough University, Leicestershire, LE11 3TU, UK

*Author for correspondence: Email: g.l.witcomb@lboro.ac.uk

Received date: June 02, 2020 Accepted date: July 17, 2020

Copyright: $\odot 2020$ McLeod CJ, et al. This is an open-access article distributed under the terms of the Creative Commons Attribution License, which permits unrestricted use, distribution, and reproduction in any medium, provided the original author and source are credited.
Citation: McLeod CJ, James LJ, Witcomb $\mathrm{GL}$. Food-to-mealtime associations influence food selection in a UK-based sample. Arch Nutr Food Sci 2020; 1(1):15-19.

\begin{abstract}
Objective: To explore the extent to which food-to-mealtime associations influence food choice when portion sizes are manipulated.

Methods: Participants ( $n=215)$ were shown 11 well-known foods via online questionnaire. Participants were asked about previous consumption, mealtime-appropriateness and preference to consume typical breakfast and lunch foods when portion sizes were manipulated in different mealtime scenarios.

Results: Ten out of 11 foods were considered mealtime-specific. When breakfast and lunch food portions were equal, congruous foods for the mealtime were preferred. At breakfast, smaller portions of breakfast foods were preferred to larger portions of lunch foods. However, at lunch, larger portions of breakfast foods were preferred to smaller portions of lunch foods.
\end{abstract}

Conclusions and Implications: Food choice is influenced by food-to-mealtime associations but may be moderated circumstantially by the interaction of appropriateness, perceived volume and expected satiety. Findings suggest that on-pack specification of mealtime appropriateness may increase the potential for foods to be selected.

Keywords: Mealtimes; meal planning; food selection; food choice; weight management.

\section{Introduction}

Humans tend to consume food throughout the day in a regular pattern [1]. This routinised behaviour reflects primal foraging strategies of repeating tried and tested fruitful paths to obtain food, without predation or starvation [2]. Modern-day food-related routines are still unpinned by these ancestral behaviours but are shaped by social, environmental and culture factors that influence preferences and beliefs about food $[3,4]$. Although routines may differ across the world, one pervasive element is that food tends to be consumed at specific times of day, and with specific descriptive terms; i.e., breakfast, lunch and dinner [4]. Research has shown that the majority of daily energy intake is consumed at three mealtimes throughout the day; $15-25 \%$ of daily energy intake is consumed at breakfast, $30-40 \%$ at lunch and $35-45 \%$ at dinner [5-8]. Frequent repetition of set meal timings, and the associated amount of food consumed at each mealtime, forms expectations for similar experiences in subsequent similar situations. This concept is termed 'associative learning' and is an example of classical conditioning where, in the context of eating behaviour, a food or characteristic of food becomes associated with a particular stimulus through repeated exposure [9-11]. That is, when a particular food is consumed at a given mealtime, it can become associated (solely or partially) with that mealtime.

In the UK, the variety of food options at different mealtimes has increased exorbitantly since post-war rationing was lifted in the mid-20th Century. Many different types and quantities of foods are now readily available to purchase and consume throughout the day. For example, breakfast options have expanded from items such as toast, cereal or a 'full English', to more varied options such as breakfast burritos, chicken on waffles with maple syrup, or paleo, gluten-free, vegan cookie dough. This expansion of breakfast options has occurred while foods that were traditionally advertised as 
breakfast foods have had their mealtime-specific association removed from their packaging. For example, Kellogg's Corn Flakes was advertised as 'The Sunshine Breakfast' from the 1960s-1990s. Today, the packaging makes no specific reference to breakfast. With the increase in access to purchase and consume a large variety of foods throughout the day, it has been suggested that the survival-based necessity of associating particular foods with particular foraging paths (i.e., mealtimes) is redundant in the modern day and, thus, that traditional food-to-mealtime associations are becoming less clear [12].

Therefore, the current study explored whether there was evidence for strong food-to-mealtime associations in the modern day in the UK by assessing previous mealtime-specific consumption and beliefs about the appropriateness of eating particular foods at particular mealtimes. We also wanted to test the extent to which participants preferred to consume certain foods at certain mealtimes when portion sizes were manipulated. The results will show the extent to which food-to-mealtime associations interact with portion size to influence food choice. These findings are important to investigate for two specific reasons. First, to help food product manufacturers and marketers understand whether traditional food-to-mealtime associations endure in the modern day and drive food selection. Second, to provide initial evidence about whether emphasising the specific mealtime at which a food is 'meant' to be consumed may increase the potential for a food to be chosen, and to increase food intake, at a specific meal. Findings in-line with this supposition would present a beneficial strategy for increasing food intake in vulnerable populations, such as the elderly or the infirm.

\section{Methods}

\section{Overview}

Participants were asked to complete an anonymous questionnaire online which took approximately 15 minutes to complete. Participants were told that the purpose of the study was to examine thoughts and routines surrounding foods, mealtimes and portion sizes. The questionnaire was open for four months from May to September 2019. Participants were entered into a prize draw to win a $£ 25$ shopping voucher and the study received ethical approval from the Loughborough University Ethics Approvals (Human Participants) Sub-Committee.

\section{Participants}

Participants were 18 years of age and over, and were eligible to take part if they did not have 1) a vegetarian or vegan diet; 2) a diagnosed eating disorder; 3 ) a history of gastric, digestive, metabolic, cardiovascular or renal disease; 4) diabetes; or 5) photosensitive epilepsy.

\section{Measures}

Participants were asked to provide their age, sex, height $(\mathrm{kg}$ or $\mathrm{ft}$ in), body mass ( $\mathrm{kg}$ or stone and lbs), ethnicity and nationality.

Participants were asked a set of questions focussed on 11 test foods that are well-known to a UK population and traditionally consumed at breakfast and/or lunch: porridge, Cheerios, Special $\mathrm{K}$ cereal, Rice Krispies, bacon sandwich, spaghetti Bolognese, chicken salad, lasagne, chicken sandwich, fish, chips and peas, and cheese and tomato pasta. In a multiple-answer 4-point Likert matrix, participants were asked three questions for each food: 1) "At which mealtime(s) have you previously consumed each food?"; 2) "At which mealtime(s) do you think it is appropriate for you to eat each food"; and 3) "At which mealtime do you think it is generally accepted as appropriate to eat each food". The answer-options for each question were: Breakfast; Lunch; All; and, None . Dinner and snack times were not included as the aim of the study was to explore whether there was evidence for food-to-mealtime associations as a concept and traditional breakfast and lunch foods in the UK tend to differ providing an appropriate dyad to explore this aim. Participants were asked to select all answers that they felt were appropriate for each food.

Next, participants were presented with images of six test foods three traditional breakfast foods: Cheerios (Nestlé), Special K cereal (Kellogg's) and porridge (Quaker, PepsiCo) and three traditional lunch foods: Spaghetti Bolognese (Sainsbury's), Chicken salad (Sainsbury's) and Fish, chips and peas (Bird's Eye, Nomad Foods). Two questions were asked 1) "Please look at the six food options and portion sizes below carefully. Please put the foods in your order of preference to eat at 1) breakfast, to stave off hunger until lunch; and 2) lunch, to stave off hunger until dinner." For each food group, the portion size (kcal) was changed three times. Thus, participants were asked the questions above three times in total. On the first occasion, all foods were presented as $500-\mathrm{kcal}$ portions. On the second occasion, the lunch foods were $500-\mathrm{kcal}$ portions and the breakfast foods were $200-\mathrm{kcal}$ portions. On the third occasion, the lunch foods were 200-kcal portions and the breakfast foods were $500-\mathrm{kcal}$ portions. Participants were not told the energy content of the portion sizes. The images were presented to the participants in a vertical line and in a drag and drop style, allowing them to click and drag an image up and down to order their preferences. Participants were told that the top of the vertical line (position 1) was to be their most preferred option and the bottom of the vertical line (position 6) was to be their least preferred option.

\section{Data analysis}

Quantitative data was analysed descriptively using SPSS V25 (SPSS Inc.). All demographic measures are presented as means \pm SD. Responses to mealtime-specific previous consumption frequency and appropriateness questions were summarised with percentages for each level of the 4-point Likert scale. For each of the three order of preference scenarios, each of the six foods was designated a number 1 (most preferred) to 6 (least preferred) based on the frequency of responses. This data was summarised as modes to indicate the most frequent rank order of preference for each food in each scenario.

\section{Results}

\section{Participant characteristics}

Participants $(n=215)$ had an average age of $35 \pm 14 \mathrm{yr}$ (range: 19-80 yr). The sample comprised of 159 females, 54 males, one participant who preferred to self-describe and one participant who preferred not to say. Participants had an average BMI of $25.4 \pm 5.1$ $\mathrm{kg} / \mathrm{m}^{2}(\mathrm{n}=213)$ and were predominantly of white ethnicity $(91 \%)$ and British (80\%) $(\mathrm{n}=215)$.

\section{Order of preference task}

When all six test foods were equal portions $(500-\mathrm{kcal})$, the order of preference matched the expected association of the food to the mealtime (Figure 1). That is, the three breakfast foods (porridge, Special K and Cheerios) were the preferred foods to eat at breakfast 


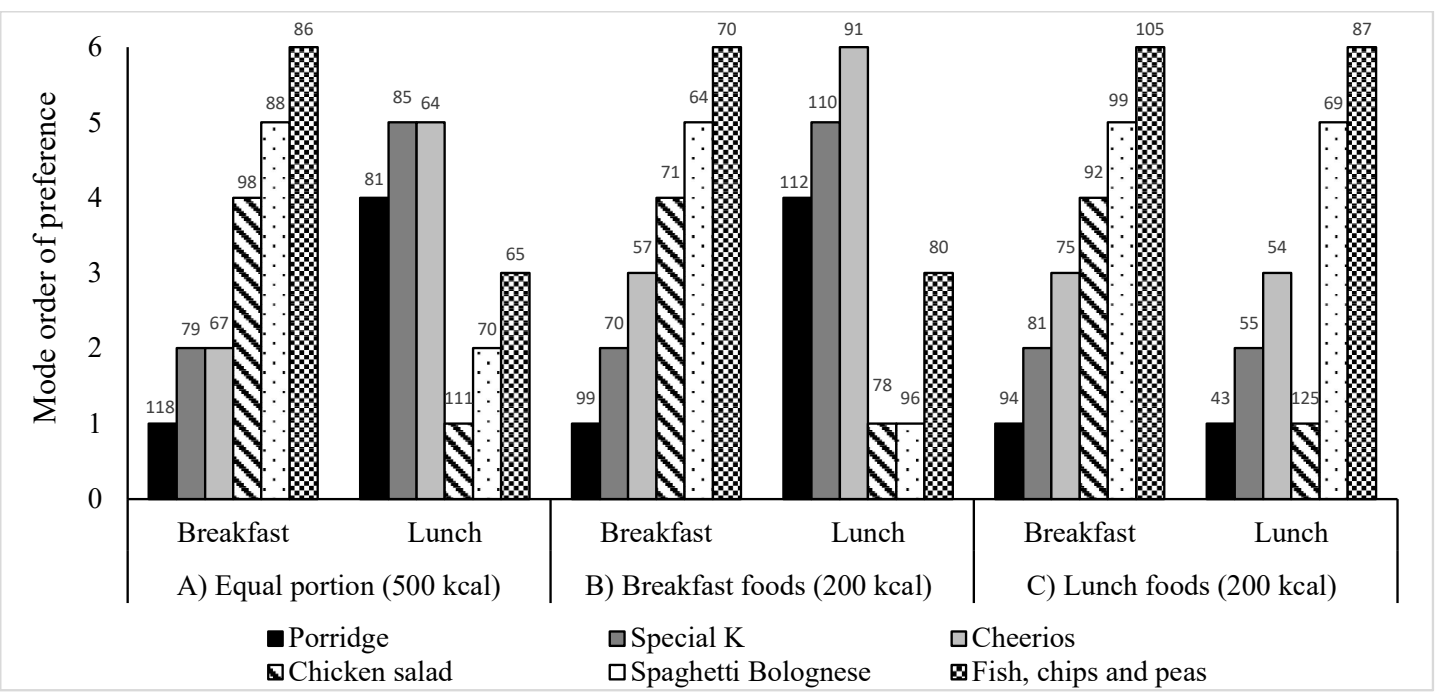

Figure 1: Modes of participants' order of preference ( $1=$ most preferred; $6=$ least preferred) for six test foods when $\mathbf{A})$ all portions were equal ( 500 kcal); B) breakfast foods (porridge, Special K and Cheerios) were 200-kcal portions (the lunch foods were 500-kcal portions); and C) when lunch foods (chicken salad, spaghetti Bolognese and fish, chips and peas) were 200-kcal portions (the breakfast foods were 500-kcal portions). Bars are presented with the number of participants who comprised the mode order of preference.

and the three lunch foods (chicken salad, spaghetti Bolognese and fish, chips and peas) were the preferred foods to eat at lunch. When the breakfast foods were presented as 200-kcal portions, and the lunch foods as $500-\mathrm{kcal}$ portions, the order of preference still matched the expected food-to-mealtime associations; the three breakfast foods were still preferred at breakfast (even though participants were shown 200-kcal portions) and the three lunch foods were preferred at lunch. When the lunch foods were presented as $200-\mathrm{kcal}$ portions and breakfast foods as $500-\mathrm{kcal}$ portions, the three breakfast foods were preferred at breakfast, as expected. However, at lunch, the 200kcal chicken salad and the 500-kcal porridge were the equally mostpreferred option. The other breakfast foods $(500 \mathrm{kcal})$ were the third and fourth most-preferred option, followed by the other lunch foods (200 kcal).

\section{Previous consumption and food-to-mealtime appropriateness}

The results show evidence for strong food-to-mealtime associations (Figure 2). That is, porridge, Special K, Cheerios and Rice Krispies are consumed by 55-69\% of participants ( $n=118-147)$ at breakfast only and are considered most appropriate to consume at this time only by the majority of participants $(60-93 \% ; n=129$ 201). In addition, $21-35 \%(n=45-75)$ of the participants considered it appropriate for them to consume these foods at both breakfast and lunch. Conversely, chicken salad, chicken sandwich, spaghetti Bolognese, cheese and tomato pasta, lasagne and fish, chips and peas are mainly consumed at lunch only $(49-84 \%$ of participants, $\mathrm{n}=105-181)$ and are predominately considered most appropriate to consume at lunch only $(63-94 \%, n=135-202)$. However, only $5-14 \%(n=10-19)$ of the participants considered it appropriate for these foods to be consumed at both breakfast and lunch, with only $1-8 \%(n=3-18)$ having actually consumed these foods at both breakfast time and lunch previously. No participants had consumed these foods or regarded them as appropriate to consume at breakfast only. Finally, Figure 2c shows that the 'both breakfast and lunch' option was the most popular response for previous consumption and appropriateness of a bacon sandwich.

\section{Discussion}

This study explored whether food-to-mealtime associations influence food choice and consumption. Our main finding was that there was strong evidence for food-to-mealtime associations for the foods used in this study and that, on the whole, congruous foods for a given mealtime were the preferred foods to consume. Congruous foods remained the preferred option at breakfast even when the portions were smaller than the portions for the incongruous foods for the given mealtime. The only exception was at lunch where larger portions of incongruous (breakfast) foods were preferred overall to smaller portions of congruous (lunch) foods.

The results revealed a clear distinction between different food-to-mealtime associations. Porridge, Special K, Cheerios and Rice Krispies were considered breakfast foods and chicken salad, chicken sandwich, spaghetti Bolognese, cheese and tomato pasta, lasagne, and fish, chips and peas were considered lunch foods. A bacon sandwich was the only food option with strong previous consumption and appropriateness associations with both breakfast and lunch. Comparing the participant responses for a bacon sandwich and a chicken sandwich is of great interest as the content of both sandwiches is very similar (meat within two pieces of bread) but the responses differed greatly regarding previous consumption and consumption appropriateness at breakfast and lunch. This highlights the process of associative learning that underpins food-to-mealtime associations. That is, the repeated exposure and reinforcement of consuming a chicken sandwich at lunch (and not breakfast), and a bacon sandwich at breakfast or lunch, forms a food-to-mealtime association and can occur notwithstanding the similarity of two foods. However, the extent to which food-to-mealtime associations are formed through explicit previous consumption of a food at a given mealtime or through the implicit learning of the perceived appropriateness of consuming a food at a given mealtime cannot be answered in this study.

Evidence of food-to-mealtime associations was also found when participants ranked their order of preference for three breakfast 

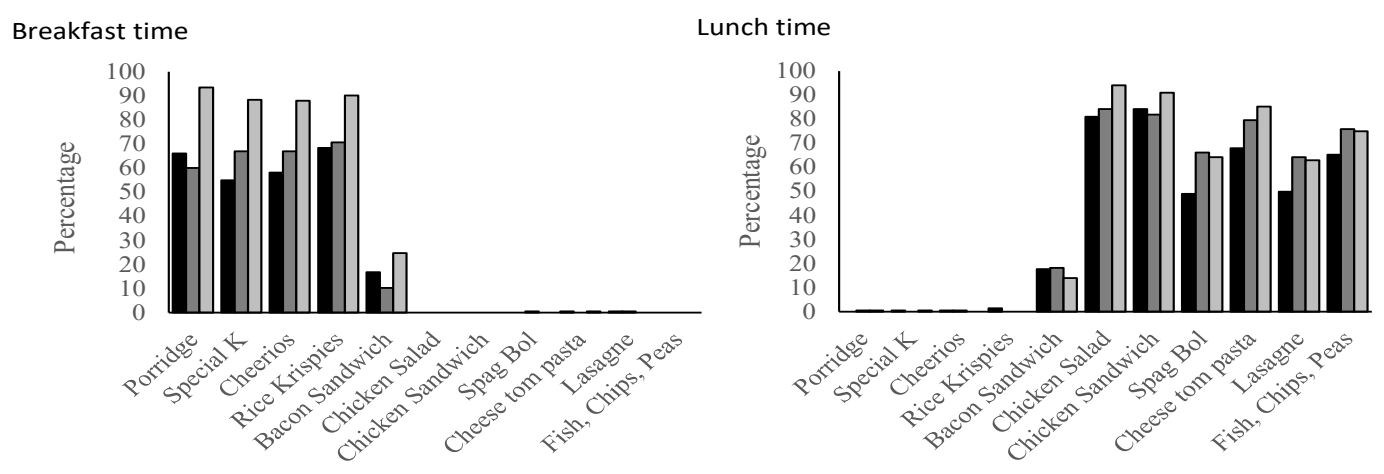

Breakfast and lunch time

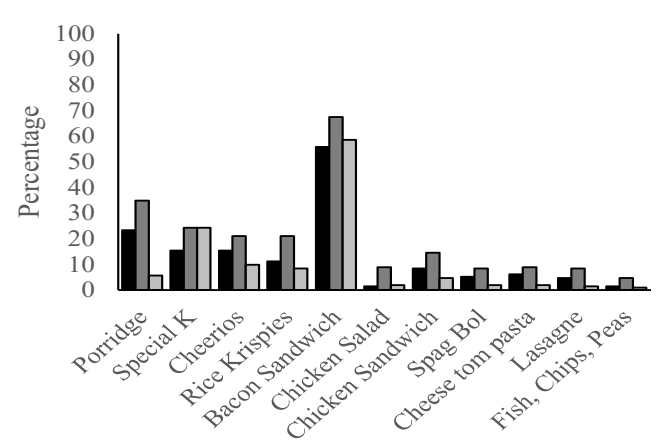

$\square$

$=$ Previously consumed
$=$ Considered appropriate for themselves to consume
$=$ Considered generally accepted as appropriate to consume

Neither breakfast nor lunch time

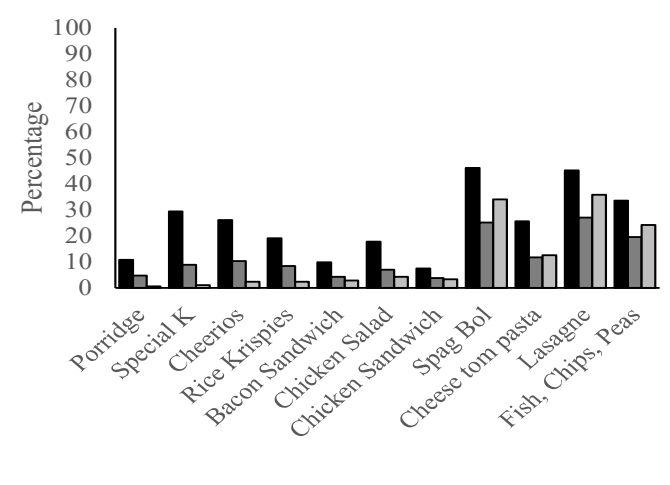

Figure 2: Participant $(n=215)$ responses showing which mealtimes a) breakfast time, b) lunch time, c) both breakfast and lunch time, d) neither breakfast nor lunch time) 1) they have consumed the test foods, 2) they believe it is appropriate for themselves to consume the test foods, and 3 ) they believe it is generally accepted as appropriate to consume the test foods.

foods and three lunch foods. When all portions were equal in energy content $(500 \mathrm{kcal})$ the breakfast foods were all preferred to the lunch foods at breakfast, and the lunch foods were all preferred to the breakfast foods at lunch. Furthermore, when the breakfast foods' portions were reduced $(200 \mathrm{kcal})$, the three breakfast foods were still preferred to the three lunch foods at breakfast time, but at lunch time, the larger portion of the lunch foods $(500 \mathrm{kcal})$ were all preferred to the smaller portions of the breakfast foods. This result highlights the influence of food-to-mealtime associations; at breakfast the smaller but mealtime-congruous food was preferred to the larger, mealtime-incongruous food. Although this finding could be explained by the wide-spread eating pattern of consuming less food at breakfast, as shown in previous research [5-8], our other results refute this suggestion. That is, when the lunch food portions were reduced $(200 \mathrm{kcal})$ at breakfast time, the larger $(500 \mathrm{kcal})$ breakfast food portions were preferred, as expected. Furthermore, in the same scenario at lunch time, all three $500-\mathrm{kcal}$ breakfast food portions were preferred to the $200-\mathrm{kcal}$ lunch food portions of spaghetti Bolognese and fish, chips and peas. Thus overall, at lunch, larger portions of incongruous foods were preferred to smaller portions of congruous foods. These results suggest that although cereals and porridge are deemed appropriate and are regularly consumed at breakfast, they are also more readily accepted at other meals, compared to lunch foods which are, in comparison, highly mealtime-specific. This finding coincides with the fact that many cereal manufacturers (e.g., Kellogg's) have removed references to breakfast on the packaging in order to remove any packagingcued mealtime restrictions. Over time, this may serve to increase the perceived acceptability of consuming cereals throughout the day, and thus may increase sales. Whether the consumer influenced the manufacturer, or vice versa, is not something that can be answered in this study.

However, it is possible that other perceptions about the portions of food presented to the participants were also considered when selecting their preferences for the six food options. For example, it is possible that the expected satiety (the perceived ability of a food or portion to stave off hunger) and/or perceived volume of the portion played a role in the decision-making process. Previous research has shown that staving off hunger until the next meal is an important consideration in pre-meal decisions about food choice and portion size [13]. The pre-consumption perception of a food's (and portion size's) ability to stave off hunger is learned from previous experience [13]. The perceived volume of the portion also plays a role in pre-meal decisions as stomach fullness, signalled by 
Citation: McLeod CJ, James LJ, Witcomb GL. Food-to-mealtime associations influence food selection in a UK-based sample. Arch Nutr Food Sci 2020; $1(1): 15-19$.

gastric stretch receptors, is a strong influence on meal termination and is also learned from previous experience $[14,15]$. Research has shown that the ability of a perceived volume of food to facilitate fullness can be estimated before eating commences and that this greatly influences food and portion selection [14]. In the current study, the perceived volume (and consequent expected satiety) of the breakfast foods and lunch foods may have influenced the order of preference. For example, the 200 -kcal chicken salad portion may have been the equally most-preferred option to eat at lunch (with the $500-\mathrm{kcal}$ porridge portion) because the perceived volume of the portion was larger (due to the meal's lower energy density) than the other two 200-kcal lunch foods (spaghetti Bolognese and fish, chips and peas). Notably, participants were not told the energy content of the portions they were asked to rank, making it more likely that the perceived volume and expected satiety of the portions played a role in these decisions. Thus, we surmise that a combination of food-tomealtime associations, perceived volume and expected satiety of the portions influenced the order in which participants preferred the six foods in the different scenarios.

\section{Implications for research and practice}

This study found evidence of strong food-to-mealtime associations in a UK-based sample and that these associations influence food choice. These findings are important for policymakers, food manufacturers and marketers as they provide evidence to suggest that traditional food-to-mealtime associations endure in the present day. However, there is also evidence that traditional food-tomealtime associations may be eroding in some instances as, at lunch, the consumption of larger portions of breakfast foods was preferred to smaller portions of lunch foods.

A clinical implication of these findings that needs to be explored in future work regards the benefit of manipulating food-to-mealtime congruency to influence energy intake. For example, as congruous foods (for the given mealtime) were the most preferred option to consume in five of our six scenarios, it should be explored as to whether food marketing that reinforces a specific mealtime at which a food is 'appropriate' to consume may help increase the potential for the food to be chosen at a specific meal. If this manipulation was successful, this novel strategy could be implemented with foods optimised in their properties to increase food intake (such as having high energy density and low expected satiety) to increase energy intake for vulnerable populations, such as the elderly or the infirm. Research should also investigate the extent to which foodto-mealtime associations are formed through mealtime-specific consumption frequency or by implicit learning of food-to-mealtime appropriateness through social cues. This is important to explore for food manufacturers and marketers to understand how novel foods can be perceived as mealtime-specific, through implicit or explicit learning, in order to facilitate a successful intervention to manipulate energy intake as presented above.

\section{Conflict of Interest}

The authors declare no conflict of interest.

\section{References}

1. Leech RM, Worsley A, Timperio A, McNaughton SA. Understanding meal patterns: definitions, methodology and impact on nutrient intake and diet quality. Nutrition research reviews. 2015 Jun;28(1):1-21.

2. Brunstrom JM, Drake ACL, Forde CG, Rogers PJ. Undervalued and ignored: Are humans poorly adapted to energy-dense foods? Appetite. 2018 Jan 1;120:589-95.

3. Warde A, Hetherington K. English households and routine food practices: a research note. The Sociological Review. 1994 Nov;42(4):758-78.

4. Jastran MM, Bisogni CA, Sobal J, Blake C, Devine CM. Eating routines. Embedded, value based, modifiable, and reflective. Appetite. 2009 Feb 1;52(1):127-36.

5. Betts JA, Richardson JD, Chowdhury EA, Holman GD, Tsintzas K, Thompson $D$. The causal role of breakfast in energy balance and health: a randomized controlled trial in lean adults. The American Journal of Clinical Nutrition. 2014 Aug 1;100(2):539-47.

6. Clayton DJ, Stensel DJ, James LJ. Effect of breakfast omission on subjective appetite, metabolism, acylated ghrelin and GLP-17-36 during rest and exercise. Nutrition. 2016 Feb 1;32(2):179-85.

7. Clayton DJ, James LJ. The effect of breakfast on appetite regulation, energy balance and exercise performance. Proceedings of the Nutrition Society. 2016 Aug;75(3):319-27.

8. Schusdziarra V, Hausmann M, Wittke C, Mittermeier J, Kellner M, Naumann A, et al. Impact of breakfast on daily energy intake-an analysis of absolute versus relative breakfast calories. Nutrition Journal. 2011 Dec;10(1):1-8.

9. Brunstrom JM. Associative learning and the control of human dietary behavior. Appetite. 2007 Jul 1;49(1):268-71.

10. Pavlov IP. Conditioned reflexes: an investigation of the physiological activity of the cerebral cortex. Oxford, Engl Oxford University Press. 1927.

11. Thibault L. Associative Learning and the Control of Food Intake. In: Dubé L, Bechara A, Dagher A, Drewnowski A, LeBel J, James P, et al., eds. Obesity Prevention: The Role of Brain and Society on Individual Behavior. Academic Press; 2010: p. 125-133.

12. Foster R, Lunn J. 40th Anniversary Briefing Paper: Food availability and our changing diet. Nutrition Bulletin. 2007 Sep;32(3):187-249.

13. McLeod CJ, James LJ, Brunstrom JM, Witcomb GL. The influence of expected satiety on portion size selection is reduced when food is presented in an 'unusual' meal context. Appetite. $2020 \mathrm{Apr}$ 1;147:104550.

14. Brunstrom JM, Collingwood J, Rogers PJ. Perceived volume, expected satiation, and the energy content of self-selected meals. Appetite. 2010 Aug 1;55(1):25-29.

15. Frayn KN. Metabolic Regulation: A Human Perspective. 3rd ed. Chichester: Wiley-Blackwell; 2010. 